\title{
A Novel Capacitance Assignment Procedure for the Design of Sensitivity- and Noise-Optimized SC-Filters
}

\author{
August Kaelin, Member, IEEE, Josef Goette, Member, IEEE, Walter Guggenbühl, Senior Member, IEEE, \\ and George S. Moschytz, Fellow, IEEE
}

\begin{abstract}
A novel procedure that determines the capacitor values for a given integrator-based $\mathrm{SC}$ network with given capacitor ratios is presented. The procedure optimally distributes a limited capacitance area among the individual circuit capacitors by minimizing the overall capacitor spread while simultaneously minimizing either sensitivity or noise. Noise in SC circuits is a function of ideal SC design parameters such as capacitor ratios and capacitance levels and of the technology-dependent parameters describing the switches and amplifiers. In our description of the noise performance, we have found a characteristic point which is only a function of $\mathrm{SC}$ design parameters and can thus serve as a measure for the noise performance. For its description a closed-form expression is used, which has the same form as the corresponding sensitivity measure. With these expressions an efficient capacitance assignment optimization procedure is derived, which is implemented in the computeraided design and optimization program package SCSYN.
\end{abstract}

\section{INTRODUCTION}

A SSUMING that the filter to be realized is specified by a given rational transfer function, the design process of a switched-capacitor (SC) filter may be divided into i) finding a suitable SC structure, ii) mapping the given polynomial coefficients onto the various capacitor ratios, which includes dynamic scaling, and iii) distributing a limited capacitance area among the individual circuit capacitors. In this contribution we concentrate on the third step: the capacitance assignment problem. We thereby restrict ourselves to the important class of twophase SC structures that usc integrators as building blocks, such as biquad cascades or ladder structures, and state the problem as follows: For an SC filter to be realized as a given integrator-based structure with given capacitor ratios, assign the fixed total capacitance to the various capacitors in the filter according to some optimization criterion. Since

Manuscript received February 28, 1990; revised March 1, 1991. This work was supported in part by the Fondation Suisse pour la Recherche en Microtechnique, under Grant 5.521.330.553/1. This paper was recommended by Associate Editor M. Ismail.

A. Kaelin and G. S. Moschytz are with the Institute for Signal and Information Processing, Swiss Federal Institute of Technology, CH-8092 Zurich, Switzerland.

W. Guggenbühl and J. Goette are with the Electronics Laboratory, Swiss Federal Institute of Technology, CH-8092 Zurich, Switzerland. IEEE Log Number 9102882. the various integrator stages can be scaled individually, the problem has $(n-1)$ degrees of freedom for an $n$ thorder filter.

The conventional approach to this problem is to assign absolute values to the individual filter capacitors by choosing the smallest capacitors of every integrator stage to be equal to some unit capacitor. The remaining capacitors are then determined by the given capacitor ratios, cf. $[1$, p. 346]. This conventional procedure does not, however, take into account the individual behavior of the integrator stages, c.g., their contributions to frequencyresponse errors and output noise. One recent approach [2] has been to optimize the noise performance of an SC biquad by using different unit capacitors for the two integrator stages. The capacitance spread is not considered. This paper solves the problem of general $n$ th-order filters (i.e., biquad and ladder networks) and shows that individual unit capacitors that are optimally adapted for each integrator stage can significantly improve both sensitivity and noise performance, while maintaining minimum capacitance spread. Thus, for example, an almost 9-dB output noise improvement was obtained in a 10th-order filter design, without increasing either the total capacitance area or the overall capacitor spread, both of which are important realizability criteria. Details of this example are discussed later in the text, where it is also shown that the improvements in noise performance directly translate into corresponding improvements in signal-to-noise ratio. It is finally noted that the problem of minimizing noise, assuming a limited capacitance area (as stated above), is equivalent to the problem of minimizing the capacitance area for a given, desired noise performance.

The outline of this paper is as follows. In Section II our model for capacitor errors and noise sources, as well as our notation, are introduced. In Section III we investigate capacitance assignments that achieve minimum capacitance spread and show that not a unique capacitance assignment, but a whole assignment region, can achieve minimum spread. This permits an additional optimization to be carried out while maintaining minimum spread. In Section IV these points are illustrated by means of a second-order example: the frequency-response error as 
well as noise is shown to be dependent on the capacitance assignment. For both performance measures, the minimum value is found to be located inside the assignment region with minimum spread. Motivated by this result, we propose an optimization strategy for general $n$ th-order filters in Section V, which minimizes frequency-response errors, or, alternatively, output noise, and simultaneously realizes the minimum overall capacitance spread. The computer-intensive sensitivity and noise computations required in Section IV reveal the need for a simplified analysis. However, whereas the frequency-response errors are computable in terms of SC design parameters only, the noise computations also involve technology-dependent parameters describing nonideal switches and amplifiers. Based on observations made for the second-order filter example of Section IV, we propose a new measure for the noise performance that depends only on SC design parameters, or in other words, is independent of technology parameters. We present an explicit closed-form expression for this noise measure that closely resembles the corresponding expression for the magnitude-response error. Using these formulas as objectivc functions, wc cxplicitly formulate the constrained optimization problem and derive an efficient algorithm for its solution in Section VI. This optimization algorithm is implemented as part of our SC-filter design program package SCSYN [3]. In Section VII, we use a 10th-order ladder filter to verify the usefulness of the optimization algorithm, and of our noise measure. We do this by comparing the sensitivity and true noise performance of our optimized design (i.e., also taking into account technology-dependent parameters) with that of the corresponding conventional design.

\section{Error Models and Notation}

Error Models for Capacitor Values: Two basically different error sources deteriorate the accuracy of capacitors in MOS integrated circuits; one is systematic, affecting all capacitors in the same way, and the other is purely random. Because the transfer functions of SC filters depend only on capacitor ratios, systematic errors that yield constant relative changes do not effect the filter performance. Since a major systematic error is caused by undercutting of capacitor plates, i.e., during etching, a properly designed capacitor has a constant area/perimeter ratio, in order to obtain constant relative changes. A standard method of achieving this is to break up larger capacitors into parallel combinations of $n$ identical small capacitors, so-called unit capacitors. Random errors are caused by global and local oxide and edge variations. According to [4], local variations generally result in errors which are uncorrelated, whereas global effects result in errors that are fully corrclated between adjacent capacitors. Thus, if the unit capacitors realizing a specific capacitor ratio are placed adjacent to each other on a chip, the errors due to global variations can be neglected.

According to [5], the remaining local errors result in a combined error for the unit capacitor $C_{0}$ described by

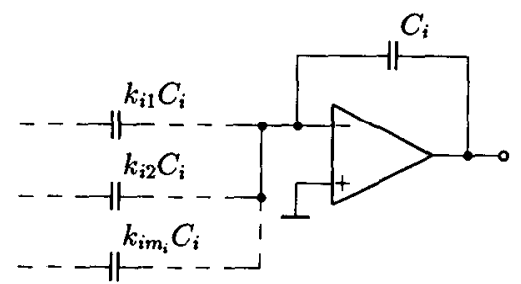

Fig. 1. Notation used for the numbering of the integrator stages and the individual capacitors of a filter.

$\sigma_{c 0}=\left(K_{\mathrm{lc}} C_{0}^{1 / 2}+K_{\mathrm{lo}} C_{0}\right)^{1 / 2}$, where $\sigma_{c 0}$ denotes the standard deviation of $C_{0}, K_{\mathrm{le}}$ the local edge effect, and $K_{\mathrm{lo}}$ the local oxide effect. For a parallel combination of $n$ unit capacitors $n C_{0} \hat{=} C_{n}$ we obtain, due to uncorrelated error terms, $\sigma_{\mathrm{cn}}=\left(K_{\mathrm{le}} n^{1 / 2} C_{n}^{1 / 2}+K_{\mathrm{lo}} C_{n}\right)^{1 / 2}$. For a given capacitor $C$, the resulting $\sigma_{c}$ is thus dependent on the chosen size of the unit capacitor $C_{0}$. In [6] it has been shown that for most commonly used technologies the dominant error term is due to local oxide effects. Noting further that $n^{1 / 4}$ is a slowly increasing function of $n$, it is reasonable to neglect the local edge effects. This leads to the following simple capacitor error model, which is independent of the chosen unit capacitor size.

Each circuit capacitor $C_{i}$ is assumed to be an independent, Gaussian distributed random variable with mean $C_{i, o}$ being the ideal capacitor-value and variance $E\left[\left(C_{i}-C_{i, o}\right)^{2}\right]$ $\hat{=} \sigma_{c, i}^{2}$. The corresponding standard deviation $\sigma_{c, i}$ is assumed to be $\sigma_{c, i}=c_{\alpha} \sqrt{C_{i, o}}$ with $c_{\alpha}$ denoting a technology constant.

Noise Models: In an SC circuit, noise is introduced by switches and amplifiers that are realized by noisy semiconductor devices. We model switches in their oN-state by finite, fixed conductances $G$ in parallel with white-noise current sources of (two-sided) spectral density $2 k T G$, where $k T$ denotes the product of Boltzmann's constant and the absolute temperature. In their off-state the switches are modeled by open circuits. The amplifiers too are assumed to introduce broadband noise, which, again, is modeled by white noise. The amplifiers are modeled by simple operational transconductance amplifiers (OTA's) consisting of a voltage-controlled current source with transconductance $g$ in parallel with a white-noise current source of spectral density $2 k T g \gamma$, with $\gamma$ denoting the noise factor of the amplifier. This simple amplifier model describes the noise adequately for our purposes at some output terminal of the SC circuit, and serves to develop the ideas leading to our proposed noise optimization.

Notation: The individual capacitors in a filter are numbered as indicated in Fig. 1. The input capacitors to the $i$ th integrator stage with integrating capacitor $C_{i}$ are denoted by $k_{i j} C_{i}$. They are connected to the surrounding circuitry by either switches or short circuits. The total capacitance of the $i$ th integrator stage is $\left(1+\Sigma_{j} k_{i j}\right) C_{i}$ and is denoted by $C_{s i}$, and the ratio $C_{s i} / C_{i}=\left(1+\sum_{i} k_{i j}\right)$ by $\beta_{i}$. The numbering used within integrator stage $i$ is such that $k_{i 1} \leqslant k_{i 2} \leqslant \cdots k_{i, m_{i}}$ if there are $m_{i}$ input capacitors in that stage, and the $n$ integrator stages of the filter are 
numbered such that stage 1 has the largest stage spread among all stages followed by the one of stage 2 , and so on. For SC filters with all capacitance ratios less than unity, this numbering scheme means that $k_{11} \leqslant k_{21} \leqslant \cdots$ $\leqslant k_{n 1}$.

\section{Capacitance Assignment and Minimal SPREAD}

For an integrator-based SC-filter the individual input capacitances $C_{i j}=k_{i j} C_{i}$ and the integrating capacitors $C_{i}$ are related to the total capacitance per stage, $C_{s i}$, by ${ }^{1}$ $C_{i j}=\left(k_{i j} / \beta_{i}\right) C_{s i}$. Since the $k_{i j}$ are fixed, the stage capacitances $C_{s i}$ uniquely determine the individual capacitances. We call the vector of stage capacitances $c_{s} \hat{=}$ $\left(C_{s 1}, C_{s 2}, \cdots, C_{s n}\right)^{T}$ the capacitance assignment. Denoting the available capacitance area for the whole filter by $C_{\text {tot }}$, we have the obvious constraint $\sum_{i} C_{s i} \leqslant C_{\text {tot }}$. We may relate $c_{s}$ to $C_{\text {tot }}$ by

$$
c_{s} \hat{=} \lambda C_{\text {tot }}
$$

where the parameter vector $\lambda \hat{=}\left(\lambda_{1}, \lambda_{2}, \cdots, \lambda_{n}\right)^{T}$ is an alternative formulation of the capacitance assignment.

Note that the constraint $\sum_{i} C_{s i} \leqslant C_{\text {tot }}$ has no influence on the capacitor spread of the filter realization. As will be shown in Section VI, the constraint always holds with equality for minimum magnitude-response errors and minimum noise. Thus the total available capacitance should be used, i.e., $\sum_{i} C_{s i}=C_{\text {tot }}$, or

$$
\sum_{i=1}^{n} \lambda_{i}=1
$$

Using the abbreviations ${ }^{2} C_{i, \max } \hat{=} \max _{j} C_{i j}$ and $k_{i, \max } \hat{=}$ $\max _{j} k_{i j}$, and correspondingly $C_{i, \min }$ and $k_{i, \min }$, the spread of stage $i$ is given by $\rho_{i} \hat{=} C_{i, \max } / C_{i, \min }=k_{i, \max } / k_{i, \min }$. Notc that, in contrast to the ovcrall circuit spread $\rho$, the stage spreads $\rho_{i}$ are independent of the assignment parameters $\lambda_{i}$ and are given by the specified capacitor ratios $k_{i j}$. With our numbering scheme, stage 1 has the largest stage spread, namely $k_{1, \max } / k_{11}$. Since this spread is fixed by the capacitor ratios $k_{1 j}$ to be realized by stage 1 , the overall spread in the circuit cannot be less than this stage spread, which then represents the minimum achievable circuit spread. The circuit spread, however, can become larger, depending on the capacitance assignment. This is the case when considering the ratio of maximum and minimum capacitors that belong to different integrator stages. Thus we obtain the conditions for minimum achievable circuit spread:

$$
C_{i, \max } \leqslant C_{1, \max }, \quad C_{i, \min } \geqslant C_{1, \min }, \quad \text { for } \quad 2 \leqslant i \leqslant n .
$$

\footnotetext{
${ }^{1}$ The notation introduced in the previous section has been generalized in the sense that each capacitor is described by two indices, with the integrating capacitors denotcd by $C_{i 0}$. In a similar way the capacitor ratios have been generalized by introducing $k_{i 0} \hat{=} 1$.

${ }^{2}$ Note that the maximum (or minimum) is taken over all capacitors in the stage, including the integrating capacitor.
}

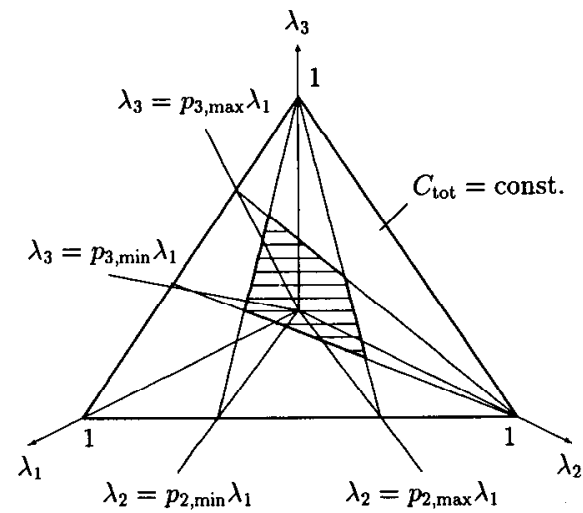

Fig. 2. Minimum spread region for a third-order filter.

With

$C_{i, \max }=\left(k_{i, \max } \lambda_{i} / \beta_{i}\right) C_{\mathrm{tot}}$ and $C_{i, \min }=\left(k_{i, \min } \lambda_{i} / \beta_{i}\right) C_{\mathrm{tot}}$ we obtain the minimum spread conditions for the assignment parameters:

$$
p_{i, \min } \lambda_{1} \leqslant \lambda_{i} \leqslant p_{i, \max } \lambda_{1}, \quad \text { for } \quad 2 \leqslant i \leqslant n
$$

where we have defined $p_{i, \text { max }} \hat{=} \beta_{i} k_{1, \text { max }} /\left(\beta_{1} k_{i, \text { max }}\right)$, and $p_{i, \min } \hat{=} \beta_{i} k_{1, \min } /\left(\beta_{1} k_{i, \min }\right)$.

Equation (3) is illustrated for a third-order example in Fig. 2. The region with constant total capacitance $C_{\text {tot }}$, or equivalently $\sum_{i} \lambda_{i}=1$, is given in a three-dimensional $\lambda$ spacc. Equation (3) defines a polyhedron achieving minimum capacitor spread, which, with our notation, is given by the spread of stage 1 . Outside this region, the spread increases " $1 / \lambda$-like" [7]; see also the illustrative example in Section IV.

The important point to note is that the minimum spread $\rho_{\min }$ is not restricted to a single value of $\lambda$ (corresponding to a single capacitance assignment), but to a range of $\lambda$ values. This permits an additional optimization such as minimum sensitivity or minimum noise to be carried out, in which case the corresponding optimum $\lambda$-value is to be found within the minimum spread range. The resulting optimized circuit is then guaranteed to have the minimum capacitance spread $\rho_{\min }$. This is illustrated by the example in the following section and discussed in detail in Section $\mathrm{V}$.

\section{An lllustrative Example}

As an illustrative example we use the biquad circuit shown in Fig. 3. With $k_{11}=k_{12}=0.0314$, and $k_{13}=k_{21}=$ 0.314 , this bandpass filter has a nominal center frequency of $100 \mathrm{kHz}$ at a clock rate of $2 \mathrm{MHz}$ and a Q-value of 10 . For the minimum achievable circuit spread we find $\rho_{\min }$ $=k_{1, \max } / k_{1, \min }=k_{10} / k_{11}=1 / k_{11} \approx 31.85$. Using (2) we replace $\lambda_{1}$ by $\lambda$ and $\lambda_{2}$ by $(1-\lambda)$. With (3), the minimum spread is found in the interval $\lambda_{l} \leqslant \lambda \leqslant \lambda_{u}$ with $\lambda_{l} \approx 0.512$, and $\lambda_{u} \approx 0.913$. In Fig. 4 , the overall spread $\rho$ as a function of the assignment parameter $\lambda$ is shown. Outside the minimum spread range, it increases as $1 / \lambda$ and $1 /$ $(1-\lambda)$ as shown in [7]. 


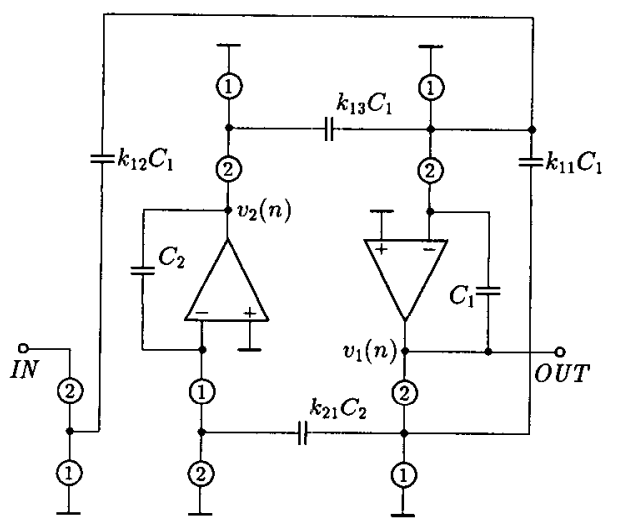

Fig. 3. Circuit diagram of second-order bandpass filter example.

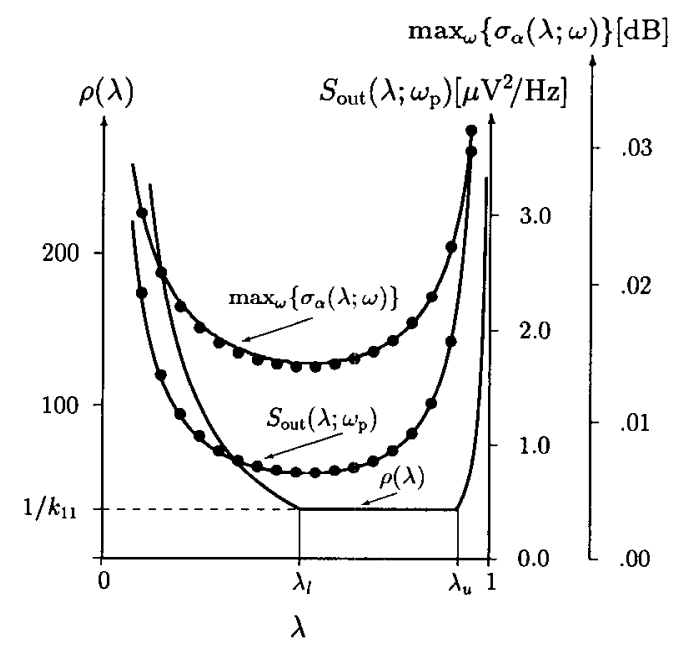

Fig. 4. Spread $\rho(\lambda)$, magnitude-response error $\max _{\omega}\left\{\sigma_{\alpha}(\lambda ; \omega)\right\}$ and noise $S_{\text {out }}\left(\lambda ; \omega_{\mathrm{p}}\right)$ as functions of the assignment $\lambda$. The dots represent numerical values computed by SCANAL and WATSCAD.

Magnitude-Response Errors: Capacitor errors introduce errors both in the magnitude- and phase-response of a given SC circuit. However, here we consider only magnitude-response errors because these are of more practical importance. Five hundred Monte Carlo simulations were carried out with the SC analysis program SCANAL [8] using our capacitor error model with $c_{\alpha}=10^{-4} \sqrt{\mathrm{p}^{5}}$ and $C_{\text {tot }}=2.69 \mathrm{pF}$. The maximum value of the standard deviation $\sigma_{\alpha}(\omega)$ of the magnitude response in the frequency range of $80 \mathrm{kHz}$ to $120 \mathrm{kHz}$ is used as a frequency independent measure for the magnitude-response error. As shown in Fig. 4, $\max _{\omega}\left\{\sigma_{\alpha}(\omega)\right\}$ is a function of the assignment parameter $\lambda$ with the minimum located within the minimum capacitance-spread interval $\left[\lambda_{l}, \lambda_{u}\right]$. Thus the filter can be designed to be optimum with respect to capacitance spread as well as to magnitude-response error.

Output Noise: Using an extended version of the WATSCAD SC-network analysis package [9], [10], the discrete noise at the node "OUT" in our second-order filter example caused by noisy switches and amplifiers has been numerically evaluated, based on the noise models described in Section II. The noise spectral density at the

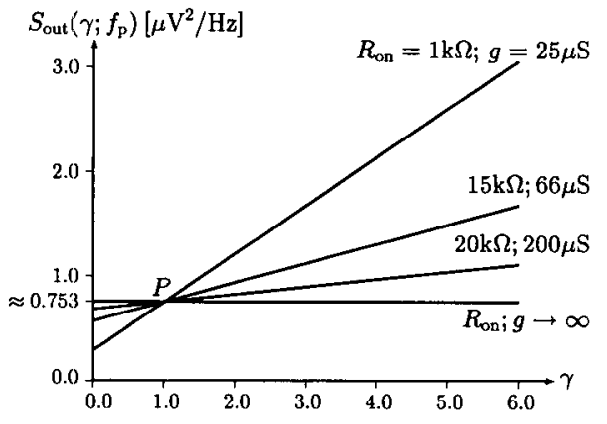

Fig. 5. Noise spectral value at center frequency as a function of the noise factor $\gamma$ for various combinations of switch oN-resistances and amplifier transconductances. The different pairs $\left(R_{\text {on }} \hat{=} 1 / G ; g\right)$ are selected to maintain approximately equal charge transfer levels.

filter's center frequency $\omega_{\mathrm{p}}, S_{\text {out }}\left(\omega_{\mathrm{p}}\right)$, is shown in Fig. 5 as a function of the amplifiers noise factor $\gamma$ for various combinations of parameters $R_{\mathrm{on}} \hat{=} 1 / G$ and $g$ and $\lambda=$ $0.5{ }^{3}$ Fig. 5 illustrates the following typical characteristics: The noise $S_{\text {out }}\left(\omega_{\mathrm{p}}\right)$ is linear in the noise factor $\gamma$ and there is a point $P$ where all the linear characteristics (which differ according to $R_{\text {on }}$ and $g$ ) meet. Such a point $P$ has been observed in all other SC-filter examples. Although a more exact analysis reveals that the linear charactcristics do not exactly meet in a point (except for undamped integrators [11]), but in a small region, it is found in the companion paper [12] that, for practical designs, this region is very small and can be approximated by a point. Furthermore, [12] shows that the magnitude of this so-called crossing point is dependent only on SC design parameters. It is therefore reasonable to use this magnitude as a measure for the true noise performance, which is independent of technology parameters. In Fig. 4, this function, $S_{\text {out }}\left(\lambda ; \omega_{\mathrm{p}}\right)$, is shown for the example at hand as evaluated by WATSCAD. It is seen that the noise measure achieves its minimum value at an assignment $\lambda$ that lies within the interval $\left[\lambda_{l}, \lambda_{u}\right]$ where minimum capacitance-spread is achieved. It is therefore possible to realize the filter in our example with a design that is optimum with respect both to spread and noise.

As this example also shows, the design optimum with respect to noisc is ncarly optimum with respect to magnitude-response errors, a behavior which has been found to be typical. Because noise is usually the critical performance measure, it is advisable to optimize a circuit with respect to noise in the knowledge that the resulting magnitude-response error will also be small.

\section{Optimum Capacitance Assignment}

\subsection{Strategy for an Optimization}

The conventional method for the computation of absolute capacitor values in a given SC-filter circuit is to choose the minimum capacitance of each stage $i, C_{i \text {, min }}$, to

\footnotetext{
${ }^{3}$ For a fair comparison, the pairs $\left(R_{\text {on }}, g\right)$ are selected such that the magnitude response errors caused by incomplete charge transfers are in the same order of magnitude for the various combinations and negligible in the present context.
} 
be equal to the unit capacitance $C_{0}$ [1]. This results in the stage capacitances $C_{s i}=\left(\beta_{i} / k_{i, \min }\right) C_{0}$. Thus a fixed total capacitance area $C_{\text {tot }}$ leads to the unit capacitance $C_{0}=$ $C_{\text {tot }} / \sum_{i} \beta_{i} / k_{i, \min }$. Since $C_{i, \min }=C_{0}$ for all stages $i$, we obtain $\lambda_{i}=p_{i, \min } \lambda_{1}$, for $2 \leqslant i \leqslant n$. In view of (2) this results in the following assignment parameters for the conventional method:

$$
\tilde{\lambda}_{i}=p_{i, \min } /\left(1+\sum_{j=2}^{n} p_{j, \min }\right), \quad \text { for } \quad 2 \leqslant i \leqslant n .
$$

In our second-order example, with $\lambda_{1}$ and $\lambda_{2}$ replaced by $\lambda$ and $1-\lambda$, respectively, we obtain $\tilde{\lambda}=\lambda_{u}$, which is the upper bound of the minimum spread region as shown in Fig. 4. However, this figure shows that a capacitance assignment that minimizes either magnitude-response errors or noise, say $\lambda^{*}$, could considerably improve the corresponding performance, compared to the conventional assignment $\tilde{\lambda}=\lambda_{u}$. We emphasize that $\tilde{\lambda}$ and $\lambda^{*}$ require the same total capacitance $C_{\text {tot }}$, and realize the same minimum capacitor spread $1 / k_{11}$.

In general, the optimum assignment vector $\lambda^{*}$ may not lie within the minimum-spread region. As mentioned earlier, outside the minimum-spread region the spread grows very fast, which means that for a given $C_{\text {tot }}$, assignments outside this minimum-spread region lead to some capacitors that are extremely small. As is well known, this situation is to be avoided for practical reasons. It is therefore reasonable to limit the capacitance assignments to the minimum-spread region specified in the $\lambda$-space by (3). Thus we can formulate the optimization problem as follows: Find the assignment $\lambda^{*}$ that minimizes either magnitude-response error or noise and simultaneously achieves a minimum overall capacitor spread.

In order to obtain an efficient optimization procedure, we next present explicit closed-form expressions for the magnitude-response error and the noise measure introduced above, which avoid computer-intensive evaluations as performed for the illustrative example in Section IV. The optimization procedure itself is developed in Section VI.

\subsection{Magnitude-Response Errors}

Seeking an analytical expression for the magnituderesponse error that permits efficient computation, we apply a first-order approximation, $\Delta \alpha$, to the amplituderesponse error. Thus we use only the linear terms of the corresponding Taylor-series expansion with respect to the capacitance ratio errors $\Delta k_{i j}$ :

$$
\Delta \alpha(\omega)=\sum_{i=1}^{n} \sum_{j=1}^{m_{i}} \frac{\partial \alpha_{o}(\omega)}{\partial k_{i j}} \Delta k_{i j}
$$

where $\partial \alpha_{o}(\omega) / \partial k_{i j}$ denotes the partial derivative of the magnitude response $\alpha(\omega)$ evaluated at the nominal $k_{i j}$. In the above equation we again use a first-order approximation for $\Delta k_{i j}$; with $k_{i j}=C_{i j} / C_{i}$, and hence $\Delta k_{i j}=1 / C_{i}$.

$$
\begin{aligned}
& \left(\Delta C_{i j}-k_{i j} \Delta C_{i}\right) \text {, we obtain } \\
& \Delta \alpha(\omega)=\sum_{i=1}^{n}\left\{\sum_{j=1}^{m_{i}} \frac{\partial \alpha_{o}(\omega)}{\partial k_{i j}} \Delta C_{i j}\right. \\
& \left.-\left(\sum_{j=1}^{m_{i}} \frac{\partial \alpha_{o}(\omega)}{\partial k_{i j}} k_{i j}\right) \Delta C_{i}\right\} \frac{1}{C_{i}} .
\end{aligned}
$$

Using our capacitance-error model (random, independent capacitance errors $\Delta C_{i j}$ with standard deviation $\sigma_{c_{i j}}=$ $c_{\alpha} \sqrt{C_{i j}}$ ), we find for the variance of the magnituderesponse error $E\left[(\Delta \alpha)^{2}\right] \hat{=} \sigma_{\alpha}^{2}$ :

$$
\begin{aligned}
\sigma_{\alpha}^{2}(\omega)=\sum_{i=1}^{n}\left\{\sum_{j=1}^{m_{i}}\left(\frac{\partial \alpha_{o}(\omega)}{\partial k_{i j}}\right)^{2} k_{i j}\right. \\
\left.+\left(\sum_{j=1}^{m_{i}} \frac{\partial \alpha_{o}(\omega)}{\partial k_{i j}} k_{i j}\right)^{2}\right\} \frac{c_{\alpha}^{2}}{C_{i}} .
\end{aligned}
$$

For our second-order example, the evaluation of (4) is plotted in Fig. 4. The curve actually looks like an interpolation of the simulated (dotted) values. This excellent agreement with the Monte-Carlo simulation indicates the correctness of our expression (4) for practical capacitor errors and corresponding capacitor-ratio errors which are usually on the order of less than $1 \%$. Even if larger capacitor errors are assumed (e.g., if the unit capacitors are small, or the process uses quantized values with poor capacitor matching), expression (4) is still a sufficiently good approximation because, in the considered optimization, only the assignment achieving a minimum is sought.

\subsection{The Noise Measure}

In the companion paper [12], a formula for the approximative computation of the noise measure introduced above (i.e., the magnitude of the crossing point noise) has been derived. For an $n$ th-order integrator-based SC-filter this discrete-noise (two-sided) spectral density is given by

$$
S_{n_{\text {out }}}(\omega)=\sum_{i=1}^{n}\left\{\sum_{j \in N_{i}}\left|H_{i j}\left(\exp \left(j \omega T_{s}\right)\right)\right|^{2} \frac{1}{k_{i j}}\right\} \frac{2 k T}{C_{i}} .
$$

I Iere, $n$ denotes the number of integrator stages in the filter, the set $N_{i}$ contains the indices $j$ of the switched input capacitors $C_{i j}$ of integrator stage $i$, and $H_{i j}(z)$ is the transfer function from capacitor $C_{i j}$ to the filter output. Note that, as desired, the above explicit expression for our noise measure depends only on SC design parameters, or in other words, it is independent of technology parameters. For our second-order example this expression is used in Fig. 4 to interpolate the simulated (dotted) values of $S_{\text {out }}\left(\lambda, \omega_{\mathrm{p}}\right)$. The excellent agreement with the WATSCAD simulation indicates the correctness of (5). Excellent agreement has also been found for higher order filters as reported in [12].

Referring to step ii) of the filter design procedure outlined in Section I, we note that the capacitor ratios are usually obtained by a scaling process which causes the signal levels of all filter nodes to be maximum (scaled for 
maximum undistorted output). It is important to note that this scaling process not only maximizes the achievable output swing, but simultaneously minimizes our noise measure (5) for a given capacitance assignment. A detailed discussion of this interesting fact is given in Appendix A which, besides proving the statement, makes clear that the result is not at all obvious. We conclude that an SC circuit scaled for maximum undistorted output achieves a maximum signal-to-noise ratio if the given capacitance area is distributed such as to minimize our noise measure.

\section{Optimization Procedure}

\subsection{Problem Formulation}

As motivated by the discussion in Section 5.1, we consider only those designs as "good" that achieve the minimum possible capacitance spread. Thus, we restrict ourselves to the region in the $\lambda$-space that is given by (3). Within this $\lambda$-region, either the magnitude-response error (4), or our noise measure (5), may be minimized. With $C_{i}=\left(\lambda_{i} / \beta_{i}\right) C_{\mathrm{tot}}$, the objective function for each case can be written in the form

$$
f(\lambda ; \omega)=\sum_{i=1}^{n} \frac{a_{i}(\omega)}{\lambda_{i}} .
$$

The frequency dependence may be climinated by usc of $F(\lambda) \hat{=} \max _{\omega \in \Omega} f(\lambda ; \omega)$, which is useful for the magnitude-response error, or by $F(\lambda) \hat{\wedge} \int_{\omega \in \Omega} f(\lambda ; \omega) d \omega$, which is useful for our noise measure with $F(\lambda)$ corresponding to the noise power in the frequency range $\Omega$. In this way, the frequency-dependent coefficients $a_{i}(\omega)$ are transformed into positive constants $b_{i}$, and for $\lambda_{i}$ 's satisfying (3), the following constrained minimization problem can be formulated: Minimize $F(\boldsymbol{\lambda})=\sum_{i} b_{i} / \lambda_{i}$ subject to $\sum_{i} \lambda_{i}$ $=1$ and the inequality constraints $p_{i, \min } \lambda_{1} \leqslant \lambda_{i} \leqslant p_{i, \max } \lambda_{1}$ for $2 \leqslant i \leqslant n$ and $\lambda_{i}>0$ for all $i$.

The above formulation inherently proves the fact that the minimum of the objective function is obtained using the entire available capacitance $C_{\text {tot }}$, as claimed in Section III: Assume there exists a minimum of $F(\lambda)$, which uses less capacitance than $C_{\text {tot }}$ meaning $\sum_{i} \lambda_{i}<1$. Increasing any parameter $\lambda_{i}$ to utilize the entire available capacitance will decrease $F(\lambda)$ because the coefficients $b_{i}$ are positive, as is seen from (4) and (5). Therefore, the minimum demands that $\sum_{i} \lambda_{i}=1$.

To emphasize that the constraints are linear in the above optimization problem we define the $n \times 1$ vector $\boldsymbol{p}_{i l}$ as

$$
p_{i l} \hat{=}(p_{i l}, 0, \cdots, \underbrace{(-1)^{l-1}}_{i \text { th place }}, 0, \cdots, 0)^{T}
$$

with $p_{i l} \hat{=} p_{i, \min }$ for $l=0$ and $p_{i l} \hat{=}-p_{i, \text { max }}$ for $l=1$, and the $n \times 1$ vector $h \bumpeq(1,1, \cdots, 1)^{T}$, and rewrite the opti- mization problem as

$$
\begin{aligned}
\text { minimize } & F(\boldsymbol{\lambda}) & =\sum_{i=1}^{n} \frac{b_{i}}{\lambda_{i}} \\
\text { subject to } & \boldsymbol{h}^{T} \boldsymbol{\lambda} & =1 \\
\text { and } & \boldsymbol{p}_{i l}^{T} \boldsymbol{\lambda} & \leqslant 0, \quad 2 \leqslant i \leqslant n, \quad l \in\{0,1\} .
\end{aligned}
$$

Note that the physical constraints $\lambda_{i}>0$ for all $i$ are not included in (6) because these requirements will automatically be fulfillcd by the algorithm to be developed.

\subsection{Discussion of the Problem}

With a simple and fast algorithm in mind, we next discuss the special properties of our optimization problem (6). First, we note that we are faced with a separable programming problem, i.e., $F(\lambda)=\sum_{i} F_{i}\left(\lambda_{i}\right)$. Consequently, the gradient vector of our objective function $F(\boldsymbol{\lambda})$ is given by $\nabla F(\boldsymbol{\lambda})=\left(-b_{1} / \lambda_{1}^{2},-b_{2} / \lambda_{2}^{2}, \cdots\right.$, $\left.-b_{n} / \lambda_{n}^{2}\right)^{T}$ and the Hessian matrix by $\nabla^{2} F(\boldsymbol{\lambda})=$ $2 \operatorname{diag}\left\{b_{1} / \lambda_{1}^{3}, b_{2} / \lambda_{2}^{3}, \cdots, b_{n} / \lambda_{n}^{3}\right\}$. Thus $\nabla^{2} F(\boldsymbol{\lambda})$ is positive definite for every relevant point $\lambda$ because $1>\lambda_{i}>0$ and $b_{i}>0$ for all $i$, meaning that $F(\boldsymbol{\lambda})$ is convex. Together with the linear equality and inequality constraints (6b) and (6c) which define a polyhedron, our problem is a convex programming problem with the fundamental property that any local minimum is a global minimum. Furthermore, since $\nabla^{2} F(\lambda)$ is strictly positive definite, the obtained minimum $\lambda^{*}$ is also unique, cf., [13, p. 257].

Denoting by $\boldsymbol{A}$ the set of those inequality constraints that are active (i.e., that hold with equality) at the minimum $\lambda^{*}$, the first-order necessary conditions for our problem (6), the so-called Kuhn-Tucker conditions, cf., [14, pp. 314 ff., p. 327], are found to be

$$
\begin{aligned}
& \nabla F\left(\boldsymbol{\lambda}^{*}\right)+\sum_{i l \in A} \mu_{i} \boldsymbol{p}_{i l}+\mu_{1} \boldsymbol{h}=0 \\
& \boldsymbol{h}^{T} \boldsymbol{\lambda}^{*}=1 \\
& \boldsymbol{p}_{i l}^{T} \boldsymbol{\lambda}^{*}=0, \quad i l \in A \\
& \boldsymbol{p}_{i l}^{T} \boldsymbol{\lambda}^{*}<0, \quad \text { il } \notin \boldsymbol{A} \\
& \mu_{i} \geqslant 0, \quad i l \in A .
\end{aligned}
$$

Note that (7a), (7b), and (7c) are the necessary conditions for the equality-constrained problem that result from the objective function (6a) together with the equality constraint (6b), and the active constraints of (6c). To verify that $A$ contains the proper set of active constraints, (7d) ensures that $\lambda^{*}$ also fulfills the inactive inequality constraints. A point $\lambda^{*}$ that fulfills all constraints is called feasible. Violating (7d) indicates that further constraints must be made active to guarantee that $\lambda^{*}$ is located within the feasible assignment region. Finally, (7e) ensures that removing a constraint from $\boldsymbol{A}$ does not further decrease the objective function

If the set $\boldsymbol{A}$ were known a priori, we would merely have io solve an equality constrained problem. Because we do not know $\boldsymbol{A}$, however, a solution procedure for (6) consists of two components: i) an algorithm for finding the correct set of active inequality constraints, and ii) a vehi- 
cle to determine the solution of the corresponding equality constrained problem. For i), we use the so-called active set method, which is adequate for convex programming problems $[14, \mathrm{ch} .11 .3]$. This is outlined in more detail in Section 6.3. Because ii) has to be solved several times in the course of the active set algorithm, we take advantage of the simple structure of our objective function and the linear constraints, and derive a closed-form solution for the equality-constrained problem; see Section 6.4 .

\subsection{Active Set Method}

The idea of an algorithm based on the active set method is to define a set of active constraints at each step, termed a working set $\boldsymbol{W}$. The working set $\boldsymbol{W}$, a subset of the given inequality constraints (6c), defines the socalled working surface $S$ by the $\lambda$-values that fulfill $p_{i l}^{T} \lambda=$ 0 for $i l \in W^{4}{ }^{4}$ Since the constraints are linear, the surface $S$ in our convex program is a hyperplane. On this hyperplane, the algorithm searches for the point $\boldsymbol{\lambda}$ which has a minimum objective function. If this point is not feasible, constraints are added to the working set $\boldsymbol{W}$ until a feasible minimum is obtained. If, at this stage, the removal of a constraint from the current working set allows a further decrease of the objective function, the algorithm continues with the search on the hyperplane given by the new reduced working set. Otherwise the algorithm terminates.

Since the number of different working sets $W$ is finite, the algorithm terminates in a finite number of steps if $a$ decrease of the objective function is guaranteed from step to step. This decrease of $F(\lambda)$ is a key point of the algorithm since, otherwise, the same working set $\boldsymbol{W}$ could be chosen a second time, causing the algorithm to cycle. As will be shown for the convex program at hand, a proper choice of the working set $W$ will decrease the objective function at each step. This guarantees the successful termination of the algorithm.

To be more precise, assume that step $(k-1)$ has obtained the feasible point $\lambda_{W_{k-1}}$ on the hyperplane $S_{k-1}$ defined by the working set $\boldsymbol{W}_{k-1}$. Further assume that in step $k$ the new working set $\boldsymbol{W}_{k}$ is obtained from $\boldsymbol{W}_{k-1}$ by either dropping a constraint or by adding a new constraint. ${ }^{5}$ The search on the hyperplane $S_{k}$ then corresponds to the following equality-constrained problem:

$$
\begin{array}{rlrl}
\text { minimize } & & F(\boldsymbol{\lambda}) & =\sum_{i=1}^{n} \frac{b_{i}}{\lambda_{i}} \\
\text { subject to } & \boldsymbol{h}^{T} \boldsymbol{\lambda} & =1 \\
\text { and } & \boldsymbol{p}_{i l}^{T} \boldsymbol{\lambda} & =0, \quad i l \in \boldsymbol{W}_{k}
\end{array}
$$

for which the solution is denoted by $\lambda_{W_{k}}^{*}$. On the working

\footnotetext{
${ }^{4}$ Note that constraints $i l$ for $l=0$ and $l=1$ are related to the lower and upper bound of the assignment parameter $\lambda_{1}$, respectively, and can, therefore, not be active simultaneously. Thus at most one of the indices $i 0$ and $i 1$ can be in the set $W$.

${ }^{5}$ Note the feasible point $\lambda_{W_{k-1}}$ belongs to both hyperplanes $S_{k-1}$ and $S_{k}$ defined by $\boldsymbol{W}_{k-1}$ and $\boldsymbol{W}_{k}$, respectively.
}

hyperplane $S_{k}$ the obtained minimum may, or may not, be feasible.

If $\lambda_{W_{k}}^{*}$ is not feasible, meaning that $\boldsymbol{p}_{i l}^{T} \lambda_{\boldsymbol{W}_{k}}^{*}>0$ for one of the free constraints $i l \notin \boldsymbol{W}_{k}$, we proceed by modifying $\lambda_{\boldsymbol{W}_{k}}^{*}$ to a feasible point $\boldsymbol{\lambda}_{\boldsymbol{W}_{k}}$ that fulfills $F\left(\boldsymbol{\lambda}_{\boldsymbol{W}_{k}}\right)<F\left(\boldsymbol{\lambda}_{\boldsymbol{W}_{k-1}}\right)$. Since $F(\cdot)$ is strictly convex, this can be done in the same way as is normally done in quadratic programming, cf., $[14$, p. 425]. The idea is to move as far as possible on the line from $\boldsymbol{\lambda}_{W_{k-1}}$ to $\lambda_{W_{k}}^{*}$ while maintaining feasibility. The feasible boundary point denoted by $\boldsymbol{\lambda}_{W_{k}}$ decreases the objective function: $F\left(\lambda_{W_{k}}\right)<F\left(\lambda_{W_{k-1}}\right)$. To show this we first note that $\lambda_{W_{k}}^{*}$ is different from $\lambda_{W_{k-1}}$ because otherwise $\lambda_{W_{k}}^{*}$ would be feasible, violating the present assumption. Since $F(\cdot)$ is strictly convex, it follows that $F\left(\lambda_{W_{k}}^{*}\right)<$ $F\left(\lambda_{W_{k}}\right)<F\left(\lambda_{W_{k-1}}\right)$ as long as ${ }^{6} \lambda_{W_{k}} \neq \lambda_{W_{k-1}}$. With $\boldsymbol{d}_{k} \stackrel{\lambda_{k}^{k}}{=} \lambda_{W_{k}}^{*}$ $-\boldsymbol{\lambda}_{\boldsymbol{W}_{k-1}}$ we writc for the improved point $\boldsymbol{\lambda}_{\boldsymbol{W}_{k}}=\boldsymbol{\lambda}_{\boldsymbol{W}_{k-1}}+$ $\alpha_{k} \boldsymbol{d}_{k}$, with $\alpha \in(0,1]$ as large as possible while maintaining feasibility: $\boldsymbol{p}_{i l}^{T} \boldsymbol{\lambda}_{\boldsymbol{W}_{k}} \leqslant 0$, for $i l \notin \boldsymbol{W}_{\boldsymbol{k}}$. This leads to

$$
\alpha_{k}=\min _{i l \notin \boldsymbol{W}_{k}, \boldsymbol{p}_{i l}^{T} \boldsymbol{d}_{k}>0}\left\{1, \frac{-\boldsymbol{p}_{i l}^{T} \boldsymbol{\lambda}_{W_{k-1}}}{\boldsymbol{p}_{i l}^{T} \boldsymbol{d}_{k}}\right\} .
$$

If $\alpha_{k}<1$ is found, a new constraint $i l$ becomes active and has to be added to $\boldsymbol{W}_{k}$ leading to the new working set $\boldsymbol{W}_{k+1}$. By contrast $\boldsymbol{\alpha}_{k}=1$ means that $\boldsymbol{\lambda}_{\boldsymbol{W}_{k}}=\boldsymbol{\lambda}_{\boldsymbol{W}_{k}}^{*}$ which leads to the second situation, meaning that $\lambda_{W_{k}}^{*}$ is feasible.

With $\lambda_{W_{k}}^{*}$ being feasible, we have to decide whether dropping an active constraint may further decrease the objective function $F(\cdot)$. The solution $\lambda_{W_{k}}^{*}$ of the equality constrained problem (8) is given by the following firstorder condition

$$
\nabla F\left(\lambda_{W_{k}}^{*}\right)+\sum_{i l \in W_{k}} \mu_{i} p_{i l}+\mu_{1} h=0 .
$$

If the Lagrange multipliers $\mu_{i}$ in (10) are nonnegative for all $i l \in \boldsymbol{W}_{k}$, then, with $\boldsymbol{\lambda}_{\boldsymbol{W}^{*}}^{*}$, our working set $\boldsymbol{W}_{k}$ fulfills the Kuhn-Tucker conditions (7), and therefore solves the convex program (6). On the other hand, if a specific Lagrange multiplier $\mu_{j}$ with $j l \in \boldsymbol{W}_{k}$ is negative, $F(\cdot)$ can further be decreased by dropping the corresponding constraint $j l$. To verify this, we relax constraint $j l$, obtaining the new working set $\boldsymbol{W}_{k+1}$, and the corresponding hyperplane $S_{k+1}$. Since our constraint vectors $\boldsymbol{p}_{i l}$ are linearly independent, there exists a $\Delta \boldsymbol{\lambda}_{j}$ for a move on $S_{k+1}, \bar{\lambda}_{\boldsymbol{W}_{k+1}} \hat{=} \boldsymbol{\lambda}_{\boldsymbol{W}_{k}^{*}}+\Delta \boldsymbol{\lambda}_{j}$, such that

$$
\boldsymbol{p}_{i l}^{T} \overline{\boldsymbol{\lambda}}_{\boldsymbol{W}_{k+1}}=\boldsymbol{p}_{i l}^{T} \Delta \boldsymbol{\lambda}_{j}= \begin{cases}c_{j}, & \text { for } i=j, i l \in \boldsymbol{W}_{k} \\ 0, & \text { for } i \neq j, i l \in \boldsymbol{W}_{k}\end{cases}
$$

because $\boldsymbol{p}_{i l}^{T} \boldsymbol{\lambda}_{\boldsymbol{W}_{k}}^{*}=\mathbf{0}$ for the active constraints (il $\in \boldsymbol{W}_{k}$ ). Note that, except for constraint $j l$, all other constraints of $\boldsymbol{W}_{k}$ are still active in $\boldsymbol{W}_{k+1}$. It can easily be shown (cf., [14, ch. 10.7]) that the move with $\Delta \boldsymbol{\lambda}_{j}$ on this new

\footnotetext{
${ }^{6}$ The degenerated case $\lambda_{W_{k}}=\lambda_{W_{k-1}}$ is not interesting because herc the new constraint is just added, but no move is performed in the $\lambda$-space. This is not considered to be an optimization step, meaning the present step number is still $(k-1)$.
} 
hyperplane $S_{k+1}$ yields $\Delta F\left(\lambda_{\boldsymbol{W}_{k}}^{*}\right)=-\mu_{j} c_{j}$. Therefore, choosing a $\Delta \boldsymbol{\lambda}_{j}$ that satisfies (11) such that a negative $c_{j}$ results (as is needed for feasibility) will decrease the objective function $F(\cdot)$ if and only if $\mu_{j}<0$. 'This proves the claim that dropping a constraint decreases $F(\cdot)$.

What we now need for the final formulation of our optimization algorithm is the solution of (8), which consists of closed-form expressions for the minimum point $\lambda_{W_{k}}^{*}$ and for the corresponding Lagrange multipliers $\mu_{i}$ of (10).

\subsection{Analytic Solution of the Equality Constrained Problem}

For notational simplicity, we drop the step index $k$ in the following presentation of the general closed-form solution for the equality constrained problem (8). This solution is derived by first reformulating the original problem by eliminating the equality constraints $(8 \mathrm{c})$. This leads to a similar problem with a reduced set of variables, which can be solved in a straightforward manner. A back transformation to the original problem finally produccs the desired solution. Leaving the details to Appendix B, we thus find for the components of the minimum point $\lambda_{W}^{*}$ :

$$
\lambda_{W, i}^{*}=\rho_{W, i}^{*} / \sum_{k=1}^{n} \rho_{W, k}^{*}, \quad 1 \leqslant i \leqslant n
$$

where the terms $\rho_{W, \mathrm{i}}^{*}$ are given by

$$
\rho_{\boldsymbol{W}, i}^{*}= \begin{cases}1, & \text { for } i=1 \\ \sqrt{b_{i}} H(\boldsymbol{W}), & \text { for } i \neq 1, i l \notin \boldsymbol{W} \\ p_{i l}, & \text { for } i l \in \boldsymbol{W}\end{cases}
$$

and where the auxiliary expression $H(\boldsymbol{W})$ is defined as

$$
H(\boldsymbol{W})=\sqrt{\frac{1+\sum_{i l \in W}(-1)^{l} p_{i l}}{b_{1}+\sum_{i l \in W}(-1)^{\prime} \frac{b_{i}}{p_{i l}}}} .
$$

The required Lagrange multipliers $\mu_{i}, i l \in W$ are also determined in Appendix B. Since only the signs of these Lagrange multipliers are needed to decide whether a constraint $i l$ has to be dropped or not, it is sufficient to use the normalized Lagrange multipliers $\tilde{\mu}_{i} \hat{=} \mu_{i} \lambda_{W, 1}^{* 2}$, given with (B.10) by

$$
\tilde{\mu}_{i}=(-1)^{l}\left(\frac{1}{H(\boldsymbol{W})^{2}}-\frac{b_{i}}{p_{i l}^{2}}\right), \quad i l \in \boldsymbol{W} .
$$

\subsection{Optimization Algorithm}

With the expressions (9), (12), (13), (14), and (15) we are ready to formulate the optimization algorithm for the convex program (6). This algorithm consists of a main procedure Minimize shown in Fig. 6, and the two auxiliary procedures FEASIBLE and MINIMAL given in Figs. 7 and 8 , respectively. Here we use a programming-like language with self-explanatory structure elements; com-

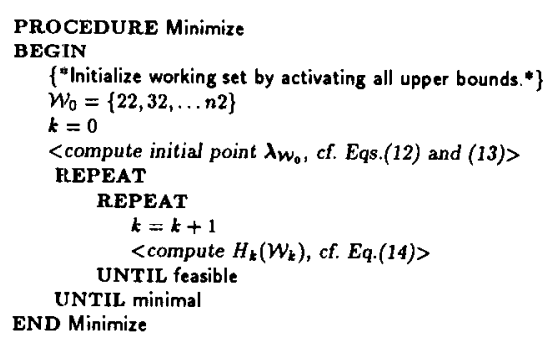

Fig. 6. Procedure Minimize.

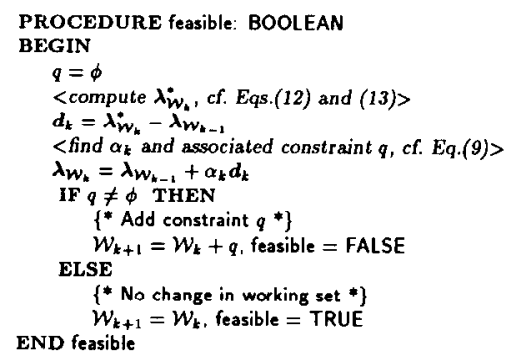

Fig. 7. Procedure FEAsible.

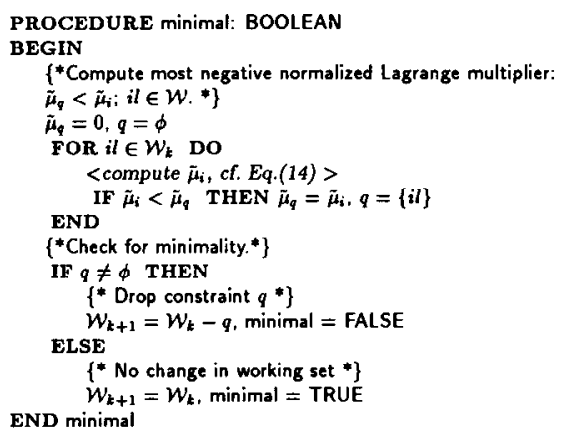

Fig. 8. Procedure Minimal.

ments are given in $\left\{*^{*}\right\}$-brackets, and "pseudo"-statements use \langle\rangle -brackets. The above algorithm has been implemented in the design program SCSYN [3].

\section{A Design Example}

As an example of practical relevance we consider the design optimization of a bandpass filter of order 10 with specifications as given in [15]. These specifications definc a passband extending from $1.2 \mathrm{kHz}$ to $1.54 \mathrm{kHz}$, a passband ripple of $0.2 \mathrm{~dB}$, and a maximum pole $\mathrm{Q}$ of 48 . Using the programs FILSYN [16], and LADNET and SCSYN [3], an SC ladder filter was generated that uses 50 $\mathrm{kHz}$ as clock frequency. The SC circuit was derived exactly from the corresponding $z$-domain transfer function, cf., [17], which, in turn, was obtained by FILSYN by prewarping and bilinearly transforming the corresponding $s$-domain transfer function. The SC circuit is shown Fig. 9. It is noted that the corresponding capacitor ratios were obtained by scaling the circuit for maximum undistorted output in the passband. In comparing different capacitance assignments, we assume a given total capacitor area of $100 \mathrm{pF}$. The magnitude response error is assumed to be 


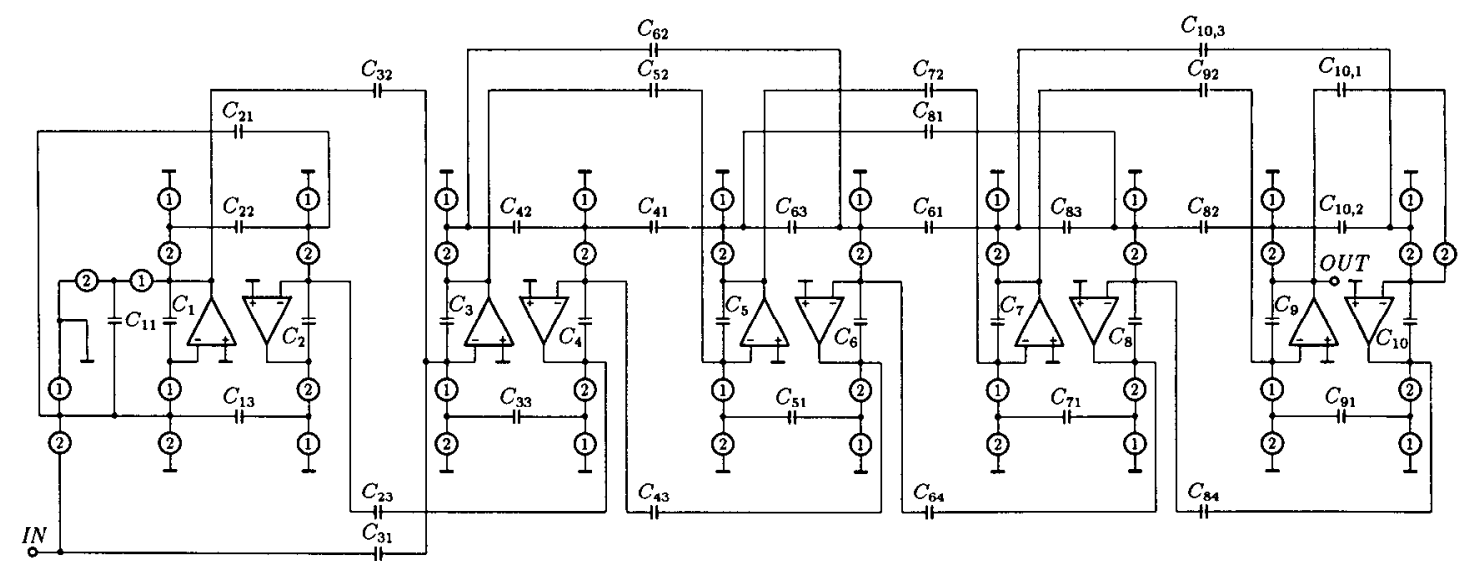

$\begin{array}{llllllllll}k_{11}=0.0324 & k_{21}=0.0039 & k_{31}=0.0028 & k_{41}=0.1365 & k_{51}=0.1977 & k_{61}=0.0321 & k_{71}=0.2081 & k_{81}=0.0349 & k_{91}=0.2848 & k_{10,1}=0.0658 \\ k_{12}=0.0547 & k_{22}=0.1436 & k_{32}=0.1026 & k_{42}=0.2433 & k_{52}=0.4093 & k_{62}=0.0874 & k_{72}=0.4118 & k_{82}=0.0417 & k_{92}=1.2547 & k_{10,2}=0.0836 \\ k_{13}=0.2066 & k_{23}=0.2679 & k_{33}=0.1646 & k_{43}=0.6392 & & k_{63}=0.1803 & & k_{83}=0.1786 & & k_{10,3}=0.0893\end{array}$

Fig. 9. Circuit diagram of 10th-order bandpass ladder filter.

TABLE I

Global versus Constrained Optimization

\begin{tabular}{lccc}
\hline $\begin{array}{c}\text { Minimum } \\
\text { criterion }\end{array}$ & Spread & $\begin{array}{c}\text { MR-error } \\
(\mathrm{dB})\end{array}$ & $\begin{array}{c}\text { Noise } \\
\left(\mu \mathrm{V}^{2}\right)\end{array}$ \\
\hline MR-error, global & 4236 & 0.074 & 333 \\
MR-error, constrained & 362 & 0.082 & 354 \\
Noise, global & 1462 & 0.081 & 270 \\
Noise, constrained & 362 & 0.087 & 293 \\
\hline
\end{tabular}

critical at the upper passband edge, at $1.54 \mathrm{kHz}$, where also the discrete-time noise spectral density of our noise mcasure is cvaluated. Thus the magnitude-response crror $\sigma_{\alpha}$, as well as the noise variance $\sigma_{n_{\text {out }}}^{2}$ in a $1-\mathrm{Hz}$ band, both at $1.54 \mathrm{kHz}$, are taken as performance measures, which are computed by our formulas (4) and (5), respectively.

In Table I we compare unconstrained-optimized (globally optimized) designs with constrained-optimized designs, that is, designs with capacitance assignments achieving the minimum overall capacitor spread, which, in our example, is 362 . If the magnitude response error is taken as the optimization criterion, we see that an unconstrained optimization can decrease the error by only about $10 \%$ compared to the corresponding constrained optimization, whereas the spread increases by a factor of almost 12. Likewise, we find with the minimum noise criterion that an unconstrained optimization can decrease the output noise by less than $8 \%$, whereas the spread is increased by a factor of 4 . This example is typical in that it demonstrates the desirability (and feasibility) of restricting the optimization to designs that result in a minimum capacitance spread.

Next, we compare these minimal spread designs ${ }^{7}$ with the conventional solution discussed in Section III, which assumes the same minimum capacitance value $C_{i 1}$ for

\footnotetext{
${ }^{7}$ The resulting magnitude-response error-optimal capacitance assignment is $\lambda=(0.0666,0.1163,0.1460,0.1383,0.1813,0.1930,0.0265$ $0.0714,0.0191,0.0416)^{T}$, whereas the noise-optimal assignment is $\lambda=$ $(0.0366,0.0942,0.1183,0.1320,0.1497,0.1563,0.0655,0.1500,0.0234$, $0.0741)^{T}$.
}

TABLE II

Standard Assignments versus Optimum Assignments. SPREAD IS 362

\begin{tabular}{lcc}
\hline Assignment & $\begin{array}{c}\text { MR-error } \\
(\mathrm{dB})\end{array}$ & $\begin{array}{c}\text { Noise } \\
\left(\mu \mathrm{V}^{2}\right)\end{array}$ \\
\hline Standard & 0.226 & 2263 \\
MR-optimal & 0.082 & 354 \\
Noise-optimal & 0.087 & 293 \\
\hline
\end{tabular}

each integrator stage. It is seen from Table II that the output noise variance $\sigma_{n_{\text {out }}}^{2}$ of our circuit is improved by almost $9 \mathrm{~dB}$ compared with that of the conventional design. Note that such improvements are also observed if the noise is taken in larger frequency bands; thus, for example, from $0.1 \mathrm{kHz}$ to $10 \mathrm{kHz}$, an improvement of 8.1 $\mathrm{dB}$ is obtained. Using the magnitude error criterion similar improvements result. It should be emphasized that all three designs feature the same overall capacitor spread of 362 , and the same total capacitor area of $100 \mathrm{pF}$. The difference between the two approaches is that in the conventional design the individual capacitors consist of multiples of a unit-capacitor that is equal to the minimum capacitance occurring in the filter (i.e., $C_{11}$ ), whereas in the improved designs each integrator stage incorporates optimally adapted unit-capacitors.

Note that in contrast to our noise measure, which is dependent only on SC design parameters, and is used in the synthesis context (computer-efficient optimization), the true noise performance is also dependent on technology parameters (noise factor $\gamma$, amplifier transconductance $g$, and switch conductance $G$ ). To verify our final designs, we compared the true noise performance of the

\footnotetext{
${ }^{8}$ The transconductance resistance combinations used are selected such that the magnitude-response errors caused by charge transfer errors are in the same order of magnitude as the presently treated magnituderesponse errors, which are due to inexact capacitances - that is, they are less than about $0.08 \mathrm{~dB}$. The required analysis was carried out using the program SWAP [18].
} 


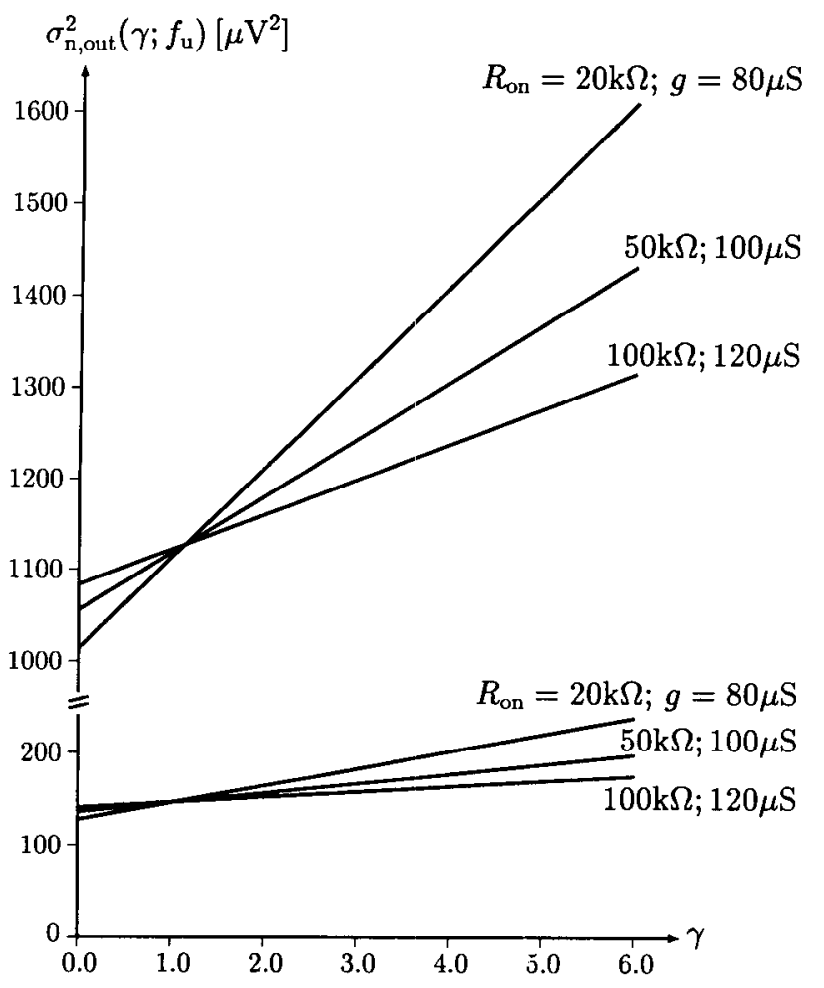

Fig. 10. True noise performance of 10th-order bandpass ladder filter for various combinations of switch on-resistances and amplifier transconductances. Noise in a $1-\mathrm{Hz}$ band at upper passband edge $f_{\mathrm{u}}=1.54 \mathrm{kHz}$. The upper curves are related to the conventional design, the lower curves to the noise-optimized design.

constrained noise-optimal design with the conventional design. For this comparison we rely on WATSCAD noise computations, which have been shown to agree well with measured results [9]. In Fig. 10, this comparison is illustrated for the noise variance in the $1-\mathrm{Hz}$ band at 1.54 $\mathrm{kHz}$, for various transconductance and switch oN-resistance combinations. ${ }^{8}$ It is seen that not only the crossing point noise (our noise measure) is far superior in the design optimized according to our procedure, but also that the true noise performance is significantly improved compared to the conventional design; the linear noise characteristics are much flatter in the optimized design, which excludes the possibility that the standard design could become superior in true noise performance for a noise factor $\gamma$ larger than that of the crossing point. Note that these relations are true not only for the illustrated noise variance, but over the whole frequency band. Because similar results have been obtained for numerous other examples, we are confident that the crossing point noise is a very useful measure for the noise performance of SC filters. In a broader context, the above results suggest the following design strategy in order to optimize the crucial noise performance: Using our procedure, choose the capacitance assignment that minimizes the technology-independent crossing point noise. Then compute the true noise performance of the obtained filter design (cf., Fig. 10) to guide a sensible design for the amplifiers and switches.

\section{Conclusions}

A design procedure for the optimum assignment of a given overall capacitance area to the various integrator stages in integrator-based SC filters is proposed. The underlying optimization criterion employed throughout is the requirement for minimum capacitance spread. This is reasonable, because we have found that minimum spread is realized in a whole assignment region, whereas unacceptably large spread-values result for assignments slightly outside this optimum region. As a result, the remaining degrees of freedom can be used for an optimization with respect to other criteria. The two criteria investigated are minimum magnitude-response error and minimum noise.

Closed-form expressions are derived that express the capacitance spread, the magnitude-response error due to capacitor errors, and the output noise as functions of the capacitance assignment. Based on these formulas, an optimization procedure is developed that either minimizes the magnitude-response errors or the noise measure, while maintaining the minimum possible capacitance spread. This procedure is implemented in the SC-filter design and optimization tool SCSYN [3]. This program also permits the structural optimization of biquad cascades where the same criteria for the optimization are used. Due to the relative simplicity of our formulas, it is possible to determine the best in a class of possible realizations by an exhaustive search within reasonable computation time.

While the expressions describing the capacitance spread and the sensitivity of the magnitude response to capacitor errors can readily be formulated in terms of capacitance ratios and assignment parameters, this is not possible for the noise minimization. Here, technology-dependent parameters such as switch oN-resistance and amplifier transconductance also come into the analysis. This is why it was necessary to develop a measure of noise performance that excludes these technology parameters and approximates the true noise in terms only of design parameters (such as capacitor ratios and capacitance levels). The high accuracy of the newly introduced noise measure is illustrated by various designs of a 10th-order ladder filter.

The usefulness of this noise measure, as well as of the optimization algorithm using it, has been demonstrated by comparing the sensitivity and the noise performance of the optimized designs of a 10th-order example filter with the corresponding performance measures resulting from a conventional design. We found that remarkable improvements are achievable with the new designs, e.g., a decrease of almost $9 \mathrm{~dB}$ in output noise was obtained for a filter using the same total capacitance and the same capacitance spread as the one based on the conventional design. Note that the decreasing noise directly translates into a corresponding improvement in signal-to-noise ratio, because the optimization does not change the dynamical behavior of the filter (i.e., the optimization permits the capacitor ratios to remain invariant). Finally, we note that noise-optimum designs are preferable to sensitivity-opti- 
mum designs, because the critical feature of SC filters is dynamic range and noise, and because, as we have empirically observed, the sensitivity performance of a noise optimum design is not far from its sensitivity optimum.

\section{Appendix A}

\section{Signal-to-Noise Ratio and Node Scaling}

We discuss here the changes in signal-to-noise ratios that are obtained by node scaling. Intuitively, node scaling that causes the signal levels of all filter nodes to be maximum (scaling for maximum undistorted output) improves the signal-to-noise ratio. The idea behind this is that the various signals in the circuit (for example the amplifier output voltages) are enlarged by this scaling process, while the noise contribution remains unchanged. The question concerning signal-to-noise ratio improvement by scaling for maximum undistorted output of SC filters is also treated in [1, pp. 339 ff.]. However, the simple noise models assumed there are different from our more accurate noise models in that ideal operational amplifiers and fixed noise sources at the amplifier input terminals are used in [1] to model amplifier noise. Switch noise does not seem to be taken into account at all. If more accurate noise models are assumed, it is not a priori clear whether scaling for maximum undistorted output will improve-or even deteriorate-the resulting signalto-noise ratio. Indeed, the approximate formula describing our noise measure (see Section 5.3) is seen to have equivalent noise sources which are dependent on the various capacitances. These noise sources have a variance of the form $2 k T /\left(k_{i j} C_{i}\right)$, where $C_{i}$ denotes the integrating capacitance of integrator stage $i$, and $k_{i j}$ are the capacitor ratios of the corresponding switched input capacitors. Because scaling for maximum undistorted output adjusts these ratios, the variances of the noise sources are also changed by this operation. This is in contrast to the assumption in [1], and evokes the question whether scaling for maximum undistorted output improves the signalto-noise ratio under all circumstances.

Consider the SC-filter section shown in Fig. 11(a) with the accompanying signal-flow graph representation in Fig. 11(b). Node scaling applied to integrator stage $i$ modifies the amplifier output-voltage $V_{i}(z)$ to $\hat{V}_{i}(z)$ by simultaneously multiplying the input branches to that stage $i$ by $\rho_{i}$ and dividing the branches leaving that stage by $\rho_{i}$. Scaling the node voltage $V_{j}(z)$ of the integrator stage $j$ means repeating this process with $\rho_{i}$ replaced by $\rho_{j}$. For the section shown in Fig. 11 these operations are described by

$$
\begin{gathered}
V_{i} \mapsto \hat{V}_{i}=V_{i} \rho_{i} \\
k_{j l} \mapsto \hat{k}_{j l}=k_{j l} \frac{\rho_{j}}{\rho_{i}} .
\end{gathered}
$$

Note that the branch connecting the two stages $i$ and $j$ is scaled twice, once as branch leaving stage $i$ by $1 / \rho_{i}$, and once as an input branch to stage $j$, by $\rho_{j}$. The transfer function from the node voltage $V_{i}(z)$ to the output, $H_{i}(z)$ $\hat{=} V_{\text {out }}(z) / V_{i}(z)$, is equivalent to the noise transfer func-

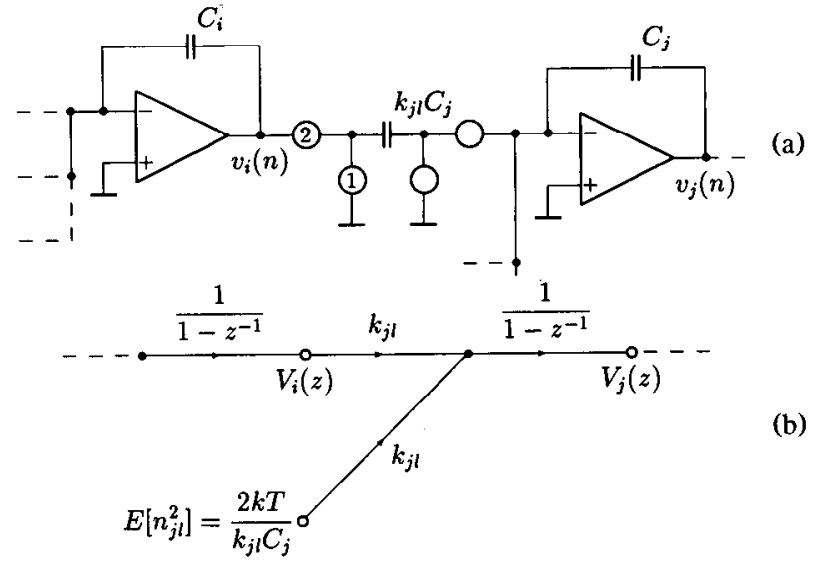

Fig. 11. SC filter section considered for signal-to-noise ratio computations.

tion from the noise source $n_{j l}$ to the output, $H_{j l}(z) \hat{=}$ $V_{\text {out }}(z) / n_{j l}(z)$, which is thus scaled to

$$
H_{j l}(z) \rightarrow \hat{H}_{j l}(z)=H_{j l}(z) \frac{1}{\rho_{i}} .
$$

Using (A.1) and (A.2), the contribution of the white noise source $n_{j l}$ with spectral magnitude $E\left[n_{j l}^{2}\right]=2 k T /\left(k_{j l} C_{j}\right)$ to the output noise spectrum, $S_{j l}(\omega)$, is seen to be scaled to

$$
\begin{aligned}
S_{j l}(\omega) \mapsto \hat{S}_{j l}(\omega) & =\left|\hat{H}_{j l}(\omega)\right|^{2} \frac{2 k T}{\hat{k}_{j l} \hat{C}_{j}} \\
& =S_{j l}(\omega) \frac{1}{\rho_{i} \rho_{j}} \frac{C_{j}}{\hat{C}_{j}}
\end{aligned}
$$

where $\hat{C}_{j}$ denotes the integrating capacitance $C_{j}$ after scaling. Clearly, the scaling operation only adjusts capacitor ratios, but says nothing about absolute capacitance values. To confine a constant total filter capacitance area, we add the requirement that the total stage capacitances remain unaltered by scaling. For integrator stage $j$ we have before scaling

$$
C_{s j}=\left(1+\sum_{l} k_{j l}\right) C_{j}
$$

and after scaling

$$
\hat{C}_{s j}=\left(1+\rho_{j} \sum_{l} \frac{k_{j l}}{\rho_{m(l)}}\right) \hat{C}_{j} .
$$

Note that as an input branch to stage $j$ the signal-flow graph branch with weight $k_{j l}$ becomes $\rho_{j} k_{j l}$ by scaling of stage $j$; as a branch connecting stage $m(l)$ to stage $j$ it becomes $k_{j l} / \rho_{m(l)}$ when scaled by $\rho_{m(l)}$. With unchanged total stage capacitances, i.e., with $C_{s j}=\hat{C}_{s j}$, the ratio $C_{j} / \hat{C}_{j}$ rcquircd in (A.3) is found using (A.4) and (A.5). The ratio of scaled to unscaled noise contribution of $n_{j l}$ 
therefore becomes

$$
\frac{\hat{S}_{j l}}{S_{j l}}=\frac{1}{\rho_{i} \rho_{j}} \frac{1+\rho_{j} \sum_{l} k_{j l} / \rho_{m(l)}}{1+\sum_{l} k_{j l}} .
$$

In the scaling process discussed here (scaling for maximum undistorted output), we must consider two separate scaling steps, that of scaling up and that of scaling down. An amplifier output-voltage $V_{i}(z)$ is scaled up if this output voltage is not as large as distortionless amplifier operation would allow. In this case, $\rho_{i}$ is larger than unity, but the maximum allowable input signal to the filter does not become larger, since the output voltage of some other amplifier would then reach its limit of distortionless operation. In order to improve the signal-to-noise ratio by scaling up, the contribution of the noise source $n_{j l}$ must decrease; this means that $\hat{S}_{j l} / S_{j l}$ must be less than unity. Assuming that in the present step only scaling up is performed, we have $\rho_{\min }=1$ from those stages that cannot be scaled up and some $\rho_{\max }>1$. Writing (A.6) as

$$
\frac{\hat{S}_{j l}}{S_{j l}}=\frac{1 /\left(\rho_{i} \rho_{j}\right)+\sum_{l} k_{j l} /\left(\rho_{i} \rho_{m(l)}\right)}{1+\sum_{l} k_{j l}}
$$

the numerator in the above formula is, with $\rho_{\min }=1$, $\rho_{\max }>1$, upper bounded by the expression in the denominator, leading to $\hat{S}_{j l} / S_{j l}<1$ as needed.

The situation where $V_{i}(z)$ is scaled down, meaning that $\rho_{i}$ is less than unity, is applied if stage $i$ limits the overall output level. Scaling down leads therefore to a larger permissible input signal to the filter. Assuming only down scaling in this step, we have $\rho_{\max }=1$ for those stages not being scaled, and a $\rho_{\min }<1$ for those stages being scaled down. Noting that $1 / \rho_{\min }$ is exactly the factor by which the maximum allowable filter input signal is being increased, the signal-to-noise ratio will be improved if the ratio $\hat{S}_{j l} / S_{j l}$ does not growing as fast as $1 / \rho_{\min }^{2}$, i.e., $\hat{S}_{j l} / S_{j l}<1 / \rho_{\min }^{2}$. This is easily seen to hold by observing that the numerator of the right hand side in (A.7) is upper bounded by $1 / \rho_{\min }^{2}$ times its denominator.

Note that a circuit with all amplifier output levels as large as distortionless operation permits, allows neither a further scaling up, nor a further scaling down step, and therefore no further improvement of the signal-to-noise ratio. We thus state the following.

Assuming noise described by (5), an integrator-based SC filter with fixed total capacitance area achieves the best obtainable signal-to-noise ratio if, and only if, it is scaled such that all its amplifier output voltages are at their maximum permissible level (scaled for maximum undistorted output).

\section{Appendix B}

Solution of the Equality-Constrained Problem

Here, we present in detail the general closed-form solution for the equality-constrained problem (8). In sub- stituting the equality constraints (8c) in (8a), we obtain the following objective function:

$$
F(\lambda)=\left(b_{1}+\sum_{i l \in W}(-1)^{l} \frac{b_{i}}{p_{i l}}\right) \frac{1}{\lambda_{1}}+\sum_{i \neq 1, i l \notin W} \frac{b_{i}}{\lambda_{i}}
$$

where the number of assignment parameters $\lambda_{i}$ has been reduced by the number of equality constraints in $(8 \mathrm{c})$ or, equivalently, by the number of active constraints $n_{c}$ in the working set $W$. Using this reduced set of variables, we reformulate (8), and obtain

$$
\begin{array}{ll}
\text { minimize } & \hat{F}(\hat{\boldsymbol{\lambda}})=\sum_{i l \notin W} \frac{\hat{b}_{i}}{\hat{\hat{\lambda}}_{i}} \\
\text { subject to } & \hat{\boldsymbol{h}}^{T} \hat{\boldsymbol{\lambda}}=1
\end{array}
$$

with

$$
\begin{aligned}
& \hat{b}_{i}=\left\{\begin{array}{cc}
\left(b_{1}+\sum_{i l \in \boldsymbol{W}}(-1)^{l} \frac{b_{i}}{p_{i l}}\right)\left(1+\sum_{i l \in \boldsymbol{W}}(-1)^{l} p_{i l}\right), \\
b_{i}, & \text { for } i \neq 1, i l \notin \boldsymbol{W}
\end{array}\right. \\
& \hat{\lambda}_{i}= \begin{cases}\lambda_{1}\left(1+\sum_{i l \in \boldsymbol{W}}(-1)^{l} p_{i l}\right), & \text { for } i=1 \\
\lambda_{i}, & \text { for } i \neq 1, i l \notin \boldsymbol{W} .\end{cases}
\end{aligned}
$$

Here the vector $\hat{\boldsymbol{h}}$ has $\left(n-n_{c}\right)$ unity components. The reduced problem (B.1) is again a convex equality-constrained program with a unique local, and therefore also global, minimum. The necessary first-order conditions for the minimum $\lambda_{W}^{*}$ are given by

$$
\begin{aligned}
\nabla \hat{F}\left(\boldsymbol{\lambda}_{W}^{*}\right)+\mu \hat{\boldsymbol{h}} & =0 \\
\hat{\boldsymbol{h}}^{T} \hat{\boldsymbol{\lambda}}_{W}^{*} & =1
\end{aligned}
$$

where $\mu$ denotes the Lagrange multiplier. For the components of the minimum point $\hat{\lambda}_{W}^{*}$, these conditions yield:

$$
\hat{\lambda}_{W, i}^{*}=\frac{\sqrt{\hat{b}_{i}}}{\sum_{j l \notin \boldsymbol{W}} \sqrt{\hat{b}_{j}}}, \quad i l \notin \boldsymbol{W} .
$$

Since any realizable assignment parameter $\lambda_{i}$ fulfills $\lambda_{i}>$ 0 , which also means $\hat{\lambda}_{i}>0$, we consider only positive square root values in (B.2).

In transforming (B.2) back to obtain the solution of the original problem (8), we use the relations

$$
\frac{\lambda_{i}}{\lambda_{1}}= \begin{cases}\frac{\hat{\lambda}_{i}}{\hat{\lambda}_{i}}\left(1+\sum_{i l \in W}(-1)^{l} p_{i l}\right), & \text { for } i \neq 1, i l \notin \boldsymbol{W} \\ p_{i l}, & \text { for } i l \in \boldsymbol{W}\end{cases}
$$

given by the expressions below (B.1) for $i \neq 1, i l \notin \boldsymbol{W}$, and by (8c) for $i l \in W$. With (B.2) these normalized $\lambda$-values yield for the minimum point $\hat{\boldsymbol{\lambda}}_{W}^{*}$ :

$$
\frac{\lambda_{W, i}^{*}}{\lambda_{W, 1}^{*}}= \begin{cases}\sqrt{b_{i}} H(\boldsymbol{W}), & \text { for } i \neq 1, i l \notin \boldsymbol{W} \\ p_{i l}, & \text { for } i l \in \boldsymbol{W}\end{cases}
$$


where the auxiliary expression $H(\boldsymbol{W})$ is defined by

$$
H(\boldsymbol{W})=\sqrt{\frac{1+\sum_{i l \in W}(-1)^{l} p_{i l}}{b_{1}+\sum_{i l \in W}(-1)^{l} \frac{b_{i}}{p_{i l}}}} .
$$

With the notation $\rho_{W, i}^{*} \hat{=} \lambda_{W, i}^{*} / \lambda_{W, 1}^{*}$, we finally obtain for the components of the minimum point $\lambda_{W}^{*}$ with (8b):

$$
\lambda_{W, i}^{*}=\rho_{W, i}^{*} / \sum_{k=1}^{n} \rho_{W, k}^{*}, \quad 1 \leqslant i \leqslant n .
$$

In the proceeding, we calculate the Lagrange multipliers $\mu_{i}$ corresponding to the active constraints (8c). First, we expand (10) in the minimum point $\lambda_{W}^{*}$, and obtain:

$$
\begin{gathered}
-\frac{b_{i}}{\lambda_{W, i}^{* 2}}+\sum_{j l \in W} \mu_{j} p_{j l}+\mu_{1}=0, \quad \text { for } i=1 \\
-\frac{b_{i}}{\lambda_{W, i}^{* 2}}+(-1)^{l-1} \mu_{i}+\mu_{1}=0, \quad \text { for } i l \in W \\
-\frac{b_{i}}{\lambda_{W, i}^{* 2}}+\mu_{1}=0, \quad \text { for } i \neq 1, i l \notin W .
\end{gathered}
$$

Note that only (B.6a) and (B.6b) are needed to determine the Lagrange multipliers $\mu_{i}$ for $i l \in W$. Using (B.6b), we obtain

$$
\mu_{i}=\left(\frac{b_{i}}{\lambda_{W, i}^{* 2}}-\mu_{1}\right)(-1)^{l-1}, \quad i l \in \boldsymbol{W}
$$

Since the active constraints fulfill

$$
\lambda_{W, i}^{*}=(-1)^{l} p_{i l} \lambda_{W, 1}^{*}, \quad i l \in W
$$

we rewrite (B.7) as

$$
\mu_{i}=\left(\frac{b_{i}}{\left(p_{i l} \lambda_{W, 1}^{*}\right)^{2}}-\mu_{1}\right)(-1)^{l-1}, \quad i l \in W
$$

and thus obtain an equation for $\mu_{1}$, if we substitute (B.8) in (B.6a):

$$
-\frac{b_{1}}{\lambda_{W, 1}^{* 2}}+\sum_{j l \in W}\left(\frac{b_{j}}{\left(p_{j l} \lambda_{W, 1}^{*}\right)^{2}}-\mu_{1}\right)(-1)^{l-1} p_{j l}+\mu_{1}=0 .
$$

Solving the above equation for $\mu_{1}$ leads, in a first step, to

$$
\mu_{1}=\frac{b_{1}+\sum_{j l \in W}(-1)^{l} \frac{b_{j}}{p_{j l}}}{1+\sum_{j l \in W}(-1)^{l} p_{j l}} \frac{1}{\lambda_{W, 1}^{* 2}}
$$

and with (B.4) to

$$
\mu_{1}=\frac{1}{\left(H(\boldsymbol{W}) \lambda_{W, 1}^{*}\right)^{2}} .
$$

Inserting (B.9) into (B.8) finally yields for the Lagrange multipliers

$$
\mu_{i}=\frac{1}{\lambda_{W, 1}^{* 2}}(-1)^{l}\left(\frac{1}{H(\boldsymbol{W})^{2}}-\frac{b_{i}}{p_{i l}^{2}}\right), \quad i l \in \boldsymbol{W} .
$$

\section{ACKNOWLEDGMENT}

The authors would like to thank Arnold Muralt for his support in carrying out various simulations.

\section{REFERENCES}

[1] R. Gregorian and G. C. Temes, Analog MOS Integrated Circuits for Signal Processing. New York: Wiley, 1986.

[2] N. Walscharts, L. Kustermans, and W. M. Sansen, "Noise optimization of switched-capacitor biquads," J. Solid-State Circuits, SC-22, pp. 445-447, June 1987.

[3] A. Kaclin and A. Muralt, LADNET and SCSYN-SC-Filter Design Tools, Reference Manual, Tech. Rep. 89 /4, Institute for Signal and Information Processing, Swiss Federal Institute of Technology, Zurich, Switzerland, 1989.

[4] J. Shyu, G. C. Temes, and F. Krummenacher, "Random error effects in matched MOS capacitors and current," J. Solid-State Circuits, vol. 19, pp. 948-955, Dec. 1984.

[5] J. Shyu, G. C. Temes, and K. Yao, "Random errors in MOS capacitors," IEEE Trans. Circuits Syst., vol. 17, pp. 1070-1076, Dec. 1982.

[6] J. McCreary, "Matching properties, and voltage and temperature dependence of MOS capacitors," J. Solid-State Circuits, vol. 16, pp. 608-616, Dec. 1981

[7] J. Goette and A. Kaelin, Criteria and Methods for the Selection of Optimized Capacitor Values in SC Filters, Tech. Rep. 88/6, Electronics Laboratory, Swiss Federal Institute of Technology, Zurich, Switzerland, 1988.

[8] R. Plodeck, U. W. Brugger, D. C. von Grucnigen, and G. S. Moschytz, "SCANAL, a program for the computer aided analysis for switched-capacitor networks," Proc. Inst. Elec. Eng., part 128-G, pp. 277-285, Dec. 1981.

[9] J. Goette and C. Gobet, "Exact noise analysis of SC circuits and a approximate computer implementation," IEEE Trans. Circuits Syst., vol. 36, pp. 508-521, Apr. 1989.

[10] M. Vlach, J. Vlach, K. Singhal, and R. Chadha, WATSCAD User's Manual, Faculty of Engineering, University of Waterloo, Waterloo, Canada, July 1983.

[11] J. Goette and W. Guggenbühl, "Noise performance of SC-integrators assuming different operational transconductance amplifier (OTA) models," IEEE Trans. Circuits Syst., vol. CAS-35, pp. 1042-1048, Aug. 1988.

[12] J. Goette, A. Kaelin, W. Guggenbühl, and G. S. Moschytz, "An approximate noise computation for general integrator-based SC filters," IEEE Trans. Circuits Syst., vol. 38, pp. 1249, Nov. 1991.

[13] P. E. Gill, W. Murray, and M. H. Wright, Practical Optimization. London: Academic, 1981.

[14] D. G. Luenberger, Linear and Nonlinear Programming. Reading, MA: Addison-Wesley, 1984.

[15] A. Kaelin, R. P. Sigg, and G. S. Moschytz, "Designing cellular parasitic-insensitive SC-ladder filters suitable for mask-programmable manufacture, Electronics Letters, vol. 22, pp. 1250-1252, Nov. 1986.

[16] G. Szentirmai, "FILSYN-A general purpose filter synthesis program," Proc. IEEE, vol. 65, pp. 1443-1458, Oct. 1977.

[17] A. Kaelin and G. S. Moschytz, "Exact design of arbitrary parasiticinsensitive elliptic SC-ladder filters in the z-domain," in Proc. IEEE Int. Symp. Circuits and Systems, pp. 2485-2488, June 1988.

[18] SWAP Switched Capacitor Simulator. Silvar Lisco, 1988. 
August Kaelin (S'83-M'91), for a photograph and biography, please see page 1254 of this issue.

Josef Goette (S'83-M'91), for a photograph and biography, please see page 1179 of the October issue of this Transactions.
Walter Guggenbühl (SM'60), for a photograph and biography, please see page 1179 of the October issue of this TRANSACTIONS.

George S. Moschytz (M'65-SM'75-F'78), for a photograph and biography, please see page 1254 of this issue. 